\title{
Efecto de la sustitución de harina de trigo con harina de avena, maíz y sorgo sobre las propiedades reológicas de la masa, texturales y sensoriales del pan
}

\author{
Effect of substitution of wheat flour with oat, corn and sorghum on \\ dough rheological, textural and sensorial bread properties
}

\author{
Francisco Vásquez Lara**凶, Samuel Verdú Amat*, Alma Rosa Islas \\ Rubio**, José Manuel Barat Baviera*, Raúl Grau Meló*, Rafael Casillas \\ Peñuelas***
}

\begin{abstract}
Vásquez, Lara, F., Verdú Amat, S., Islas Rubio, A. R., Barat Baviera, J. M., Grau Meló, R., \& Casillas Peñuelas, R. (2017). Efecto de la sustitución de harina de trigo con harina de avena, maíz y sorgo sobre las propiedades reológicas de la masa, texturales y sensoriales del pan. Investigación y Ciencia de la Universidad Autónoma de Aguascalientes, 25(71), 19-26.
\end{abstract}

\section{RESUMEN}

Se evaluó el efecto de la sustitución del 2.5, 5, 7.5 y $10 \%$ de harina de trigo (HT) con harina de avena (HA), de maíz (HM) y de sorgo (HS) sobre las propiedades reológicas de la masa, texturales y sensoriales del pan. Se determinó la capacidad de retención de agua (CRA), temperatura de empaste, viscosidad pico y viscosidad final en harinas. En masa, se determinó fuerza (F) y volumen a $1 \mathrm{~h}$ de fermentación. Se realizó un análisis sensorial en pan para determinar las preferencias de los panelistas, así como el análisis del perfil de textura (TPA). Los resultados mostraron diferencias significativas en las mediciones realizadas. El volumen de la masa obtenido al utilizar HA en los cuatro diferentes niveles no mostró diferencias con HT (136.32 $\left.\mathrm{cm}^{3} \pm 4.62\right)$. El análisis sensorial no mostró diferencias cuando el

Palabras clave: harina de trigo; harina de avena; harina de maíz; harina de sorgo; propiedades reológicas; análisis sensorial.

Keywords: wheat flour; oat; corn; sorghum; rheological properties; sensory analysis.

Recibido: 14 de septiembre de 2016, aceptado: 24 de marzo de 2017

Departamento de Tecnología de Alimentos, Universidad Politécnica de Valencia. Camino de Vera, s/n, C. P. 46022, Valencia, España. Correo electrónico: saveram@upvnet.upv.es; jmbarat@gmail.com; rgraume@ gmail.com

** Departamento de Tecnología de Alimentos de Origen Vegetal Centro de Investigación en Alimentación y Desarrollo, A. C. Carretera a La Victoria km 0.6, C. P. 83304, Hermosillo, Sonora, México. Correo electrónico: fvas@ciad.mx; aislas@ciad.mx

*** Departamento de Tecnología de Alimentos, Universidad Autónoma de Aguascalientes. Avenida Universidad 940, C. P. 20131, Aguascalientes, Aguascalientes, México. Correo electrónico: mielabejas@hotmail.com

$\triangle$ Autor para correspondencia pan se elaboró con HA o HM a $10 \%$ y el obtenido sólo de HT.

ABSTRACT

The effect of substitution of 2.5, 5, 7.5 and $10 \%$ of wheat flour $(\mathrm{HT})$ with oat flour (HA), corn (HM) and sorghum (HS) on the rheological properties of the dough, textural and sensorial bread properties was evaluated. The water holding capacity (CRA), pasting temperature, peak viscosity and final viscosity in flour was determined. In dough, force (F) and volume within an hour of fermentation was measured. A sensory analysis of bread to determine the preferences of the panelists and a texture profile analysis (TPA) were also carried out. The results showed significant differences $(p<0.05)$ among measurements. The volume of the dough using HA at the four levels did not show significant differences compared with HT (136.32 $\left.\mathrm{cm}^{3} \pm 4.62\right)$. Sensory analysis of bread showed no difference $(p>0.05)$ between the bread made with $10 \% \mathrm{HA}$ or HM and the HT only bread.

INTRODUCCIÓN

La utilización de harinas compuestas ha ido en aumento, países con problemas en la producción de trigo panadero han optado por la utilización de semillas desarrolladas localmente para elaborar harinas y ser empleadas en la sustitución de harina de trigo para la elaboración de pan. Milligan, Amlie, Reyes, Garcia, y Meyer (1981) definieron harinas compuestas como una mezcla de harinas, almidones y otros ingredientes que intentan reemplazar a la de trigo total o parcialmente en panadería o pastelería. 
IIVESTIGACIÓn Y CIERCIA DE LA UNIVERSIDAD AUTÓNOMH DE RGUASCALIERTES
20

Dentro de los granos utilizados en la sustitución de harina de trigo, se pueden mencionar leguminosas tales como la soya y garbanzo, además de otros cereales como maíz, arroz, mijo, sorgo, avena y centeno.

El uso de harinas compuestas para elaborar pan puede tener ventajas, como la disminución en el uso de la harina de trigo; por tanto, en la reducción de importación de este cereal, en los menores costos en la producción; además, al ser considerado pobre nutricionalmente, sobre todo en aminoácidos esenciales como lisina y treonina (Dhingra \& Jood, 2002), con la utilización de otros granos se podría mejorar la calidad nutricional de los productos basados en harina de trigo. Son muchas las ventajas que trae consigo una buena sustitución de éstas; desde el punto de vista nutrimental incrementa los contenidos de proteínas, aminoácidos esenciales, minerales, fibra dietaria $u$ otros nutrientes que son importantes para el buen funcionamiento de nuestro organismo. Sin embargo, es importante encontrar las proporciones adecuadas para obtener buenos parámetros de calidad, así como variables de proceso con las que se logre obtener productos agradables para el consumidor desde el punto de vista organoléptico y, por supuesto, que incrementen sus propiedades nutrimentales.

Otro punto importante es hacer consideraciones desde el punto de vista económico, ya que el precio del grano de este cereal fluctúa constantemente de acuerdo con su manejo en mercados internacionales. Es importante considerar los ahorros que podrían beneficiar al productor de harinas o al fabricante de productos basados en harinas de trigo, reemplazar una parte de ellas por otras opciones que además de sus beneficios nutrimentales, involucre un producto final de buenas características organolépticas y aceptabilidad por el consumidor y que baje los costos de las materias primas. Por lo planteado anteriormente, el objetivo del estudio fue evaluar el efecto de la sustitución de la HT con HA, HM y HS sobre las propiedades reológicas de la masa, texturales y sensoriales del pan.

\section{MATERIALES Y METODOS}

\section{Materias primas}

La HT fue obtenida de un productor local (Molí de Picó Harinas Segura, S. L., Valencia, España). La $\mathrm{HA}, \mathrm{HM}$ O HS fueron adquiridas de una tienda local (La Carabasseta), en Valencia, España. Para la panificación se utilizó aceite de girasol, levadura (Saccharomyces cerevisiae), azúcar blanca y sal marina refinada, todas ellas compradas en el mercado local. Las formulaciones que se evaluaron se basaron en la mezcla de la HA, HM O HS con la HT en las proporciones siguientes: 2.5/97.5, 5/95, 7.5/92.5 y 10/90 (peso/peso), respectivamente.

\section{Capacidad de retención de agua (CRA) de las harinas}

La CRA se realizó con base en el método 56-11 (AACC, 2000). Se colocaron $5 \pm 0.05 \mathrm{~g}$ de harina en un tubo de $50 \mathrm{ml}$ (Nalgene Centrifuge Ware) al cual se le añadieron $25 \pm 0.05 \mathrm{~g}$ de agua. La mezcla fue agitada vigorosamente por $5 \mathrm{~s}$ para hacer suspender la harina. Después se dejó reposar, agitando nuevamente hasta los $5,10,15$ y $20 \mathrm{~min}$, seguida de centrifugación por 15 min a $1000 \times g$ (Centrifuge Beckman Coulter Allegra X-30R, Brea, California, EE. UU.) a temperatura ambiente. El sobrenadante se descartó y el pellet húmedo fue decantado por 10 min para finalmente ser pesado. Los cálculos se realizaron de acuerdo a Haynes, Bettge, y Slade (2009).

\section{Análisis de viscosidad de las harinas}

El perfil de viscosidad de las harinas fue realizado de acuerdo al método 76-21 (AACC, 2000). La HT y las mezclas de la HT con HA, HM o HS fueron analizadas con base en $3.5 \pm 0.01 \mathrm{~g}$ de muestra ajustadas a $14 \%$ de humedad. La cantidad de agua a incorporar fue de $25 \pm 0.01 \mathrm{~g}$, resultado del propio ajuste al que se llevó a las harinas, como se encuentra en Shittu, Raji, y Sanni (2007). Para esta determinación se utilizó el viscosímetro RVA (Rapid Visco Analyser Super 4, Newport Scientific Australia). Mediante el software Thermocline del RVA se seleccionó el perfil de la prueba estándar 1. Los parámetros analizados fueron temperatura de empaste, viscosidad pico y viscosidad final.

\section{Determinación de fuerza ( $F)$ en la masa}

La masa utilizada para llevar a cabo la medición de F se obtuvo a partir del mixógrafo (National Mfg. Co. Lincoln, NE, EE. UU.), dicha masa se colocó en una plataforma ranurada y sobre la misma se colocó otra sin ranuras y se prensó aproximadamente $30 \mathrm{~s}$ para formar las tiras de masas, las cuales se introdujeron en una estufa (Laboratory Oven, Cole Parmer, Modelo 05015-50, Niles, Illinois, EE. UU.) a $30^{\circ} \mathrm{C}$ durante $45 \mathrm{~min}$, como se puede consultar en Ktenioudaki, O'Shea, y Gallagher (2013). Al cabo de este tiempo se midió la $\mathrm{F}$ a una velocidad de 


\section{IIVESTIGAGIÓn Y CUERCIA DE LA UกIVERSIDAD AUTÓOOOMA DE AGUASCALIERTES}

prueba de $3.3 \mathrm{mms}^{-1}$. Se realizó de acuerdo con la técnica descrita por Kieffer, Wieser, Henderson, y Graveland (1998), utilizando un analizador de textura (TA-XT2, SMS/Kieffer dough extensibility Rig Stable Micro Systems, Godalming, Surrey, Inglaterra). El analizador de textura se calibró con una pesa de $5 \mathrm{~kg}$. El umbral de la fuerza y distancia fue de $1.0 \mathrm{~g}$ y $1.0 \mathrm{~mm}$, respectivamente.

\section{Volumen de la masa}

Se midió el volumen de la masa después de 1 h de fermentación. Para ello se tomaron $70 \mathrm{~g}$ de la masa que se colocaron en un tubo cilíndrico graduado de vidrio, como en Soleimani Pour Damanab, Jafary y Rafiee (2011) (5.5 cm de diámetro y $15.5 \mathrm{~cm}$ de alto), el cual se introdujo a la cámara de fermentación (Modelo KBF720 Binder, Tuttlingen, Alemania) con humedad relativa y temperatura controlada $190 \%$ $\mathrm{HR}$ y $40^{\circ} \mathrm{C}$ ) durante $1 \mathrm{~h}$. Mediante el registro del volumen inicial de la masa y el obtenido a la hora de fermentación se calculó la capacidad de la masa para ser fermentada.

\section{Elaboración del pan}

Las proporciones de los componentes fueron de $288.3 \mathrm{~g}$ de agua, $16.9 \mathrm{~g}$ de aceite, $9.2 \mathrm{~g}$ de sal, 33.9 $\mathrm{g}$ de azúcar, $19.2 \mathrm{~g}$ de levadura y $492 \mathrm{~g}$ de la mezcla de harina $(262 \mathrm{~g}$ de harina que se mezclaron en una primera etapa por $30 \mathrm{~s}$ y $230 \mathrm{~g}$ que se adicionaron después). Para la fermentación se tomaron $250 \mathrm{~g}$ de masa que se colocaron en el molde de panificación, el cual se introdujo en la cámara de fermentación $\left(90 \%\right.$ HR y $40^{\circ} \mathrm{C}$ ) por $1 \mathrm{~h}$. Posteriormente se horneó (Modelo Fagor 2CF-3V) a $200^{\circ} \mathrm{C}$ por $15 \mathrm{~min}$.

\section{Perfil de textura (TPA) del pan}

La miga se cortó en cilindros de $4 \mathrm{~cm}$ de alto y diámetro de $3.1 \mathrm{~cm}$. Se empleó el texturómetro TA-XT Plus (Texture Analyser Stable Micro Systems, Surrey, Reino Unido). Se utilizó la celda de carga de $25 \mathrm{~kg}$ con el dispositivo TA-40 (40 mm de diámetro). La velocidad del ensayo fue de $1.7 \mathrm{mms}^{-1}$ para comprimir la miga a $50 \%$ de su altura. El tiempo entre las compresiones fue de $5 \mathrm{~s}$. Los parámetros obtenidos fueron dureza, elasticidad, cohesividad, gomosidad, masticabilidad y resiliencia, como lo que encontraron Verdú et al. (2015).

\section{Análisis sensorial del pan}

El análisis sensorial se realizó con los 13 panes elaborados, un control hecho sólo con HT y los 12 restantes elaborados con la sustitución de la
HT con HA, HM O HS en porcentajes de 2.5, 5, 7.5 y 10. Participaron 24 panelistas no entrenados que determinaron su preferencia sobre los panes elaborados con cada una de las formulaciones. Los atributos analizados fueron color, aroma, textura al tacto, apariencia, sabor, textura en boca y apreciación general. Para cada uno de los atributos se estableció una escala que fue de muy desagradable a muy agradable en la que los evaluadores señalaron su grado de preferencia.

\section{Análisis estadístico}

Los resultados se analizaron aplicando un procedimiento de comparación múltiple de medias para determinar cuáles fueron significativamente diferentes. Para discriminar entre las medias se utilizó el procedimiento de diferencia mínima significativa (LSD) de Fisher. El análisis estadístico se llevó a cabo con el programa Statgraphics Centurion XVI con un nivel de confianza de $95 \%$.

RESULTADOS

\section{Análisis en harinas}

Los resultados de CRA y el análisis de viscosidad se muestran en la figura 1 . En el caso particular de la CRA, que se observa en la figura 1 (a) se observó una tendencia a disminuir a medida que se incrementó el nivel de reemplazo, este comportamiento se presentó en los diferentes cereales utilizados en la suplencia de HT. Los valores más altos se obtuvieron cuando la HT fue sustituida por HS a 2.5 y $5 \%$ (70.61 \pm 0.14 y $71.54 \pm 0.2$ ), respectivamente, incluso mayores al obtenido en HT $(69.15 \pm 0.06)$. En lo que se refiere al análisis de viscosidad, la tendencia cuando se analizó la temperatura de empaste, que se observa en la figura 1 (b), fue de incrementar a medida que aumentó el nivel de sustitución, esto ocurrió con los tres cereales utilizados en el reemplazo de HT; sin embargo, cuando se utilizó HS a $10 \%$ se observaron los valores más altos de este parámetro $187.6^{\circ} \mathrm{C} \pm$ $0.57)$, muy por encima del obtenido por HT $\left(69.15^{\circ} \mathrm{C}\right.$ $\pm 0.64)$.

Con relación a la viscosidad pico, que se expone en la figura 1(c), aunque se observa una ligera tendencia a incrementar a medida que se aumenta el porcentaje de sustitución (HA, HM O HS), no se observaron diferencias significativas con respecto al valor obtenido en HT (2245 CP \pm 15.56), excepto cuando se utilizó HS a 2.5\% (2164 cP \pm 74.95) en la sustitución de HT. En lo que respecta a la 

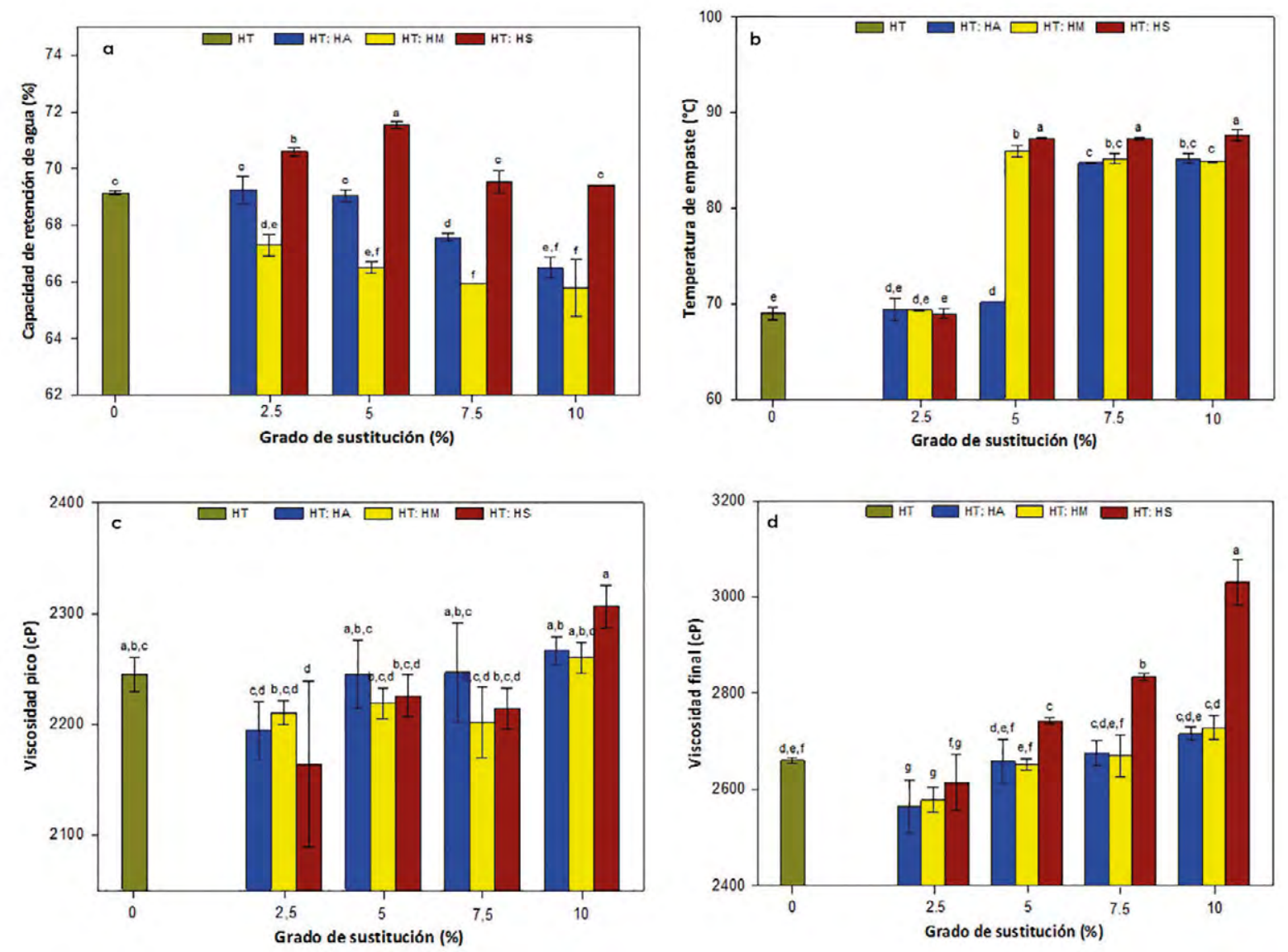

Figura 1. Capacidad de retención de agua (a), temperatura de empaste (b), viscosidad pico (c) y viscosidad final (d) en HT y en HT sustituida con HA, HM y HS en los diferentes porcentajes.

Elaboración propia.

viscosidad final que puede consultarse en la figura 1 (d), hay incrementos más pronunciados al aumentar el porcentaje de reemplazo para todos los cereales involucrados; sin embargo, estos incrementos son más evidentes cuando se utiliza HS a $5 \%$ (2742.5 cP $\pm 6.36), 7.5 \%$ (2833.5 CP \pm 6.35) o $10 \%$ (3029.5 cP \pm 47.38), y se observan diferencias significativas con respecto al valor obtenido por HT (2660 CP \pm 5.66$)$.

\section{Determinación de fuerza $(F)$ y volumen de la masa}

La figura 2 muestra los resultados de $\mathrm{F}$ y volumen de la masa a $1 \mathrm{~h}$ de fermentación. En lo que respecta a F, como se observa en la figura 2 (a), hubo diferencias significativas; una disminución importante en este parámetro fue resultado de la sustitución llevada a cabo con HM, mientras que al utilizar HA se aprecia una tendencia a disminuir al incrementar el porcentaje de reemplazo, valores menores fueron obtenidos al utilizar HA, HM y HS en la suplencia de HT con respecto al observado en HT
(0.69 N \pm 0.01 ); sin embargo, cuando se utilizó HS en la sustitución se observaron incrementos a medida que aumentó el porcentaje de reemplazo, esto fue más evidente al utilizar HS a $10 \%(0.61 \mathrm{~N} \pm 0.05)$, aun así este valor fue menor al obtenido cuando se analizó HT. En cuanto al volumen de la masa, como puede verse en la figura 2 (b), la sustitución que más benefició a este parámetro fue cuando se utilizó HA en los cuatro diferentes porcentajes, sin mostrar diferencias significativas con la masa obtenida sólo de HT (136.32 $\left.\mathrm{cm}^{3} \pm 4.62\right)$.

\section{Perfil de textura (TPA) del pan}

La tabla 1 muestra los valores de dureza (N), elasticidad, cohesividad, gomosidad, masticabilidad y resiliencia que se obtuvieron del análisis de perfil de textura del pan. Se puede apreciar que se evidenciaron diferencias significativas entre las muestras. En relación con dureza, definida como la fuerza necesaria para alcanzar la deformación máxima preestablecida, se 


\section{InVESTIGACIÓn Y GIERGIA DE LA UחIVERSIDAD AUTÓnOMA
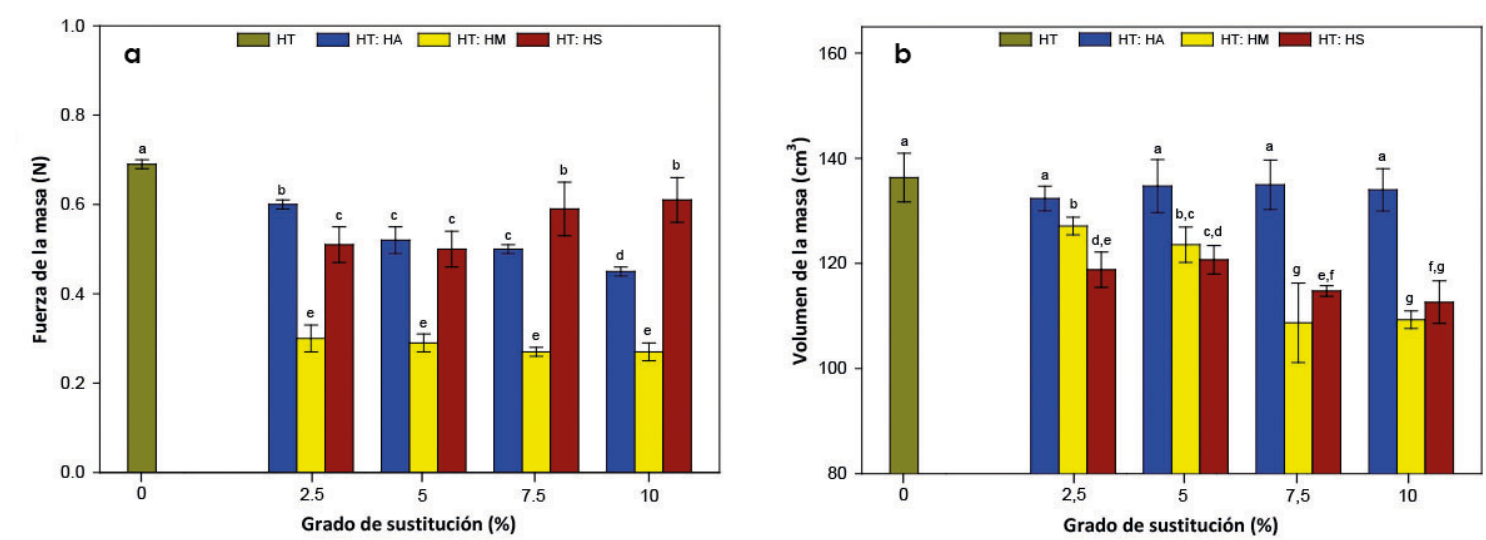

Figura 2. Fuerza de la masa (a) y volumen (b) obtenidos en HT y en HT sustituida con HA, HM y HS en los diferentes porcentajes.

Elaboración propia.

observó que la HT sustituida con sorgo en niveles de 5 (9.39 \pm 1.02$), 7.5(9.66 \pm 1.44)$ y 10\% (10.51 \pm 1.56$)$ mostró valores similares al obtenido por los panes elaborados con la harina HT (9.32 \pm 1.02$)$. El resto de las suplencias presentaron valores inferiores a los de HT. En cuanto a elasticidad, la altura registrada por el alimento cuando inició la segunda compresión con respecto a la que tenía inicialmente, no se vio afectada por el nivel de sustitución y el cereal empleado para ello, dado que no se observaron diferencias significativas.

En lo que respecta a cohesividad, relación de áreas originadas en los dos ciclos de compresión, excluyendo la zona de descompresión de cada ciclo, sí se lograron establecer diferencias significativas. Los resultados obtenidos para este parámetro están agrupados dentro de un rango de 0.78 a 0.86 . Al observar los resultados cuando la HT $(0.81 \pm 0.01)$ fue sustituida con HA, se logró apreciar diferencias significativas solamente en la sustitución de 10\% 10.83 \pm 0.01 ), mientras que al ser utilizada HM las diferencias observadas fueron cuando la $\mathrm{HT}$ fue cambiada al $5 \mathrm{y}$ $7.5 \%(0.84 \pm 0.01)$ o $10 \%(0.86 \pm 0.05)$. Cuando se utilizó $\mathrm{HS}$ en la suplencia, las diferencias significativas fueron observadas al emplear 5 o $10 \%(0.79 \pm 0.01)(0.78 \pm$ $0.01)$ con respecto al pan obtenido solamente de HT.

La gomosidad, definida como el producto de dureza por cohesividad, al igual que la dureza, cuando HT $(7.56 \pm 0.79)$ fue sustituida con HS a $5 \%$ $(7.47 \pm 0.76), 7.5 \%(7.76 \pm 1.11) \circ 10 \%(8.29 \pm 1.10)$, no se evidenciaron diferencias significativas para este parámetro. Las sustituciones con avena (HA) y maíz (HM) mostraron valores inferiores a los de HT, aquellas en las que HT fue sustituida con HA a $10 \%$ (4.03 \pm
$0.85)$ o HM a $7.5 \%$ (4.01 \pm 1.03$)$ fueron los reemplazos que presentaron menores valores. En relación con masticabilidad, definida como el producto de dureza por cohesividad por elasticidad, mostró un comportamiento muy semejante al de dureza y gomosidad, y los valores para las sustituciones con HS al $5,7.5$ y $10 \%$, iguales estadísticamente a los de HT (7.44 \pm 0.83$)$, e inferiores estadísticamente a los obtenidos por el resto de sustituciones.

Finalmente, la resiliencia fue el último de los parámetros evaluados. Al analizar el valor obtenido del pan elaborado con HT $(0.442 \pm 0.01)$ con respecto al de las suplencias con los diferentes cereales, se logró observar diferencias significativas al utilizar HA en sus diferentes niveles de sustitución, este mismo comportamiento sucedió cuando se utilizó HM, excepto al nivel de sustitución de $2.5 \%(0.446 \pm 0.01)$. Con respecto a las sustituciones donde se utilizó HS, no se observaron diferencias significativas, los valores obtenidos estuvieron por debajo (0.436 a 0.441 ) del alcanzado por HT, comportamiento opuesto al observado cuando se empleó HA en los reemplazos.

\section{Análisis sensorial del pan}

En la tabla 2 se muestran los resultados del análisis sensorial para cada uno de los panes elaborados y parámetros evaluados. En color verde se muestran aquellos panes cuyo valor no fue estadísticamente diferente del obtenido para los panes elaborados sólo con HT. En azul se muestran los panes con harina sustituida parcialmente por HA y, como se aprecia, sólo los panes con $2.5 \%$ y $5 \%$ de reemplazo presentaron diferencias en textura al tacto y apreciación general 
Tabla 1

Parámetros del análisis de perfil de textura de los panes obtenidos de HT y de HT sustituida con HA, HM y HS

\begin{tabular}{|c|c|c|c|c|c|c|}
\hline Pan & Dureza $(N)$ & Elasticidad & Cohesividad & Gomosidad & Masticabilidad & Resiliencia \\
\hline$H T$ & $9.32 \pm 1.02 \mathrm{a}$ & $0.98 \pm 0.03 a$ & $0.81 \pm 0.01$ aef & $7.56 \pm 0.79 a$ & $7.44 \pm 0.83 a$ & $0.442 \pm 0.01$ adef \\
\hline$H T: H A 2.5 \%$ & $6.38 \pm 1.04 \mathrm{~cd}$ & $0.94 \pm 0.07 a$ & $0.82 \pm 0.01$ ade & $5.22 \pm 0.8 \mathrm{~cd}$ & $4.95 \pm 0.97 \mathrm{def}$ & $0.451 \pm 0.01 b c$ \\
\hline HT: HA 5\% & $6.71 \pm 0.78 \mathrm{~cd}$ & $0.98 \pm 0.01 a$ & $0.82 \pm 0.01 \mathrm{abc}$ & $5.54 \pm 0.63 \mathrm{~cd}$ & $5.45 \pm 0.61 \mathrm{de}$ & $0.459 \pm 0.01 c$ \\
\hline HT: HA 7.5\% & $6.13 \pm 0.94 \mathrm{cde}$ & $0.97 \pm 0.08 a$ & $0.82 \pm 0.01 \mathrm{abc}$ & $5.05 \pm 0.72 \mathrm{cde}$ & $4.96 \pm 1.02 \mathrm{def}$ & $0.452 \pm 0.01 b c$ \\
\hline HT: HA $10 \%$ & $4.82 \pm 1.04$ ef & $0.92 \pm 0.11 \mathrm{a}$ & $0.83 \pm 0.01 b c$ & $4.03 \pm 0.85$ ef & $3.74 \pm 0.96 \mathrm{~g}$ & $0.455 \pm 0.01 b c$ \\
\hline$H T: H M 2.5 \%$ & $7.28 \pm 0.8 b c$ & $0.96 \pm 0.04 a$ & $0.81 \pm 0.01$ aef & $5.92 \pm 0.58 b c$ & $5.73 \pm 0.68 \mathrm{~cd}$ & $0.446 \pm 0.01$ ade \\
\hline$H T: H M 5 \%$ & $5.2 \pm 1.11$ ef & $0.95 \pm 0.05 a$ & $0.84 \pm 0.01 b$ & $4.35 \pm 0.89$ ef & $4.19 \pm 0.97 \mathrm{fg}$ & $0.452 \pm 0.01 b c$ \\
\hline HT: HM 7.5\% & $4.78 \pm 1.3 f$ & $0.94 \pm 0.06 \mathrm{a}$ & $0.84 \pm 0.01 \mathrm{~b}$ & $4.01 \pm 1.03 f$ & $3.81 \pm 1.11 \mathrm{~g}$ & $0.459 \pm 0.01 \mathrm{c}$ \\
\hline HT: HM 10\% & $5.64 \pm 0.83$ def & $0.95 \pm 0.05 a$ & $0.86 \pm 0.05 d$ & $4.82 \pm 0.65 \mathrm{de}$ & $4.62 \pm 0.76$ efg & $0.455 \pm 0.01 b c$ \\
\hline HT: HS $2.5 \%$ & $8.2 \pm 1.14 b$ & $0.98 \pm 0.02 a$ & $0.80 \pm 0.01 \mathrm{fgh}$ & $6.58 \pm 0.89 b$ & $6.49 \pm 0.94 b c$ & $0.439 \pm 0.01$ ef \\
\hline$H T: H S 5 \%$ & $9.39 \pm 1.02 a$ & $0.96 \pm 0.06 \mathrm{a}$ & $0.79 \pm 0.01 \mathrm{gh}$ & $7.47 \pm 0.76 a$ & $7.21 \pm 0.95 a b$ & $0.436 \pm 0.01 \mathrm{f}$ \\
\hline HT: HS 7.5\% & $9.66 \pm 1.44 a$ & $0.98 \pm 0.02 a$ & $0.80 \pm 0.01 \mathrm{fgh}$ & $7.76 \pm 1.11 \mathrm{a}$ & $7.61 \pm 1.04 a$ & $0.441 \pm 0.01$ def \\
\hline HT: HS $10 \%$ & $10.51 \pm 1.56 \mathrm{a}$ & $0.97 \pm 0.03 \mathrm{a}$ & $0.78 \pm 0.01 \mathrm{~h}$ & $8.29 \pm 1.1 \mathrm{a}$ & $8.08 \pm 1.17 a$ & $0.432 \pm 0.01 \mathrm{f}$ \\
\hline
\end{tabular}

Nota: $\mathrm{HT}=$ Harina trigo; $\mathrm{HA}=$ Harina avena; $\mathrm{HM}=$ Harina maíz; $\mathrm{HS}=$ Harina sorgo. $\mathrm{N}=$ Newton. El \% observado en la tabla indica el grado de sustitución de HT. Medias con letra diferente dentro de la columna expresan diferencias significativas $(p<0.05)$.

Elaboración propia.

respectivamente, en donde los valores obtenidos fueron menores al de los panes elaborados solo con HT. En amarillo se muestran las diferencias cuando la harina sustitutoria fue maíz; sin embargo, como se puede apreciar en la tabla, ningún parámetro y para ningún nivel de sustitución se presentaron diferencias significativas con los panes elaborados con HT. Finalmente, en anaranjado se muestran las diferencias cuando la harina sustitutoria fue procedente de sorgo. Aquí se aprecia que los panes elaborados con esta harina fueron los que presentaron mayores diferencias con respecto a los elaborados con HT, y en todos los casos los valores obtenidos fueron menores al de los panes elaborados solo de HT, excepto cuando se realizó la sustitución de HT al $2.5 \%$.

\section{DISCUSIÓN}

En relación con la CRA, estudios realizados por Ram, Dawar, Singh y Shoran (2005) obtuvieron valores de 53.4 a $70.6 \%$, cercano este último al obtenido en nuestro estudio para HT; observaron también que harinas con altos valores de retención de agua y fuerza de gluten intermedia son preferidas para la elaboración de un pan sin levadura consumido en la India (chapati). El tamaño de partícula es uno de los parámetros más importantes que determinan la CRA. Berton, Scher, Villieras, y Hardy (2002) observaron también que un incremento en almidón dañado podría resultar en aumentos en la CRA. En lo que respecta al incremento en la temperatura de empaste, refleja la limitación del gránulo de almidón a hincharse durante la hidratación, bajo las condiciones de calentamiento y agitación de la prueba, el incremento en enlaces disulfuro dentro de la estructura de las proteínas y la pérdida en la proporción de proteínas solubles asociadas con el almidón pueden retardar el hinchamiento del gránulo; así lo establecen Tadeu Paraginski, Levien Vanier, Berrios, Oliveira, y Cardoso Elias (2014).

La gelatinización completa es un requisito previo esencial para que el cocimiento o empaste sea observado (Batey, 2007). La reducción en el hinchamiento de los gránulos de almidón es indicativo de las bajas viscosidades pico y la disminución en la ruptura de los gránulos de almidón, esto se encuentra en Ktenioudaki et al. (2013). Los gránulos que tienen una alta capacidad de hinchamiento también tienden a tener una alta viscosidad. La velocidad de calentamiento también tiene un efecto importante sobre la viscosidad pico. Estudios realizados por Ktenioudaki et al. (2013), 
Tabla 2

Resultados del análisis sensorial realizado al pan elaborado con HT y del pan obtenido de la sustitución de HT con HA, HM y HS

\begin{tabular}{|c|c|c|c|c|c|c|c|}
\hline Pan & Color & Aroma & Textura tacto & Apariencia & Sabor & Textura boca & Apreciación general \\
\hline$H T$ & $6.48 \pm 1.39 \mathrm{a}$ & $5.81 \pm 1.59 \mathrm{ab}$ & $6.12 \pm 1.37$ a & $6.47 \pm 1.28 \mathrm{a}$ & $5.78 \pm 1.59 \mathrm{ab}$ & $4.47 \pm 1.85 a b c$ & $6.08 \pm 1.34 \mathrm{a}$ \\
\hline HT: HA $2.5 \%$ & $6.23 \pm 1.36 a$ & $5.41 \pm 1.76 b$ & $5.60 \pm 1.61 \mathrm{abc}$ & $6.38 \pm 1.41 a$ & $5.19 \pm 1.62 b c$ & $4.13 \pm 1.65 a b c$ & $5.43 \pm 1.60 \mathrm{bcd}$ \\
\hline HT: HA 5\% & $6.12 \pm 1.38 \mathrm{ab}$ & $5.63 \pm 1.48 \mathrm{ab}$ & $5.11 \pm 1.72 \mathrm{bcd}$ & $6.32 \pm 1.61 \mathrm{a}$ & $5.14 \pm 1.50 b c$ & $4.45 \pm 1.88 \mathrm{abc}$ & $5.62 \pm 1.53 \mathrm{abcd}$ \\
\hline HT: HA $7.5 \%$ & $6.21 \pm 1.48 a$ & $6.20 \pm 1.52 \mathrm{ab}$ & $5.81 \pm 1.67 \mathrm{ab}$ & $6.40 \pm 1.58 a$ & $5.16 \pm 1.45 b c$ & $4.64 \pm 2.06 a b c$ & $5.65 \pm 1.31 \mathrm{abcd}$ \\
\hline IHT: HA $10 \%$ & $6.30 \pm 1.21 \mathrm{a}$ & $5.95 \pm 1.18 \mathrm{ab}$ & $5.76 \pm 1.35 \mathrm{ab}$ & $6.22 \pm 1.75 a$ & $5.31 \pm 1.11 \mathrm{abc}$ & $3.98 \pm 1.59 b c$ & $5.56 \pm 1.25 \mathrm{abcd}$ \\
\hline$\cdot H T: H M 2.5 \%$ & $6.38 \pm 1.17 \mathrm{a}$ & $5.93 \pm 1.58 \mathrm{ab}$ & $6.26 \pm 1.34 a$ & $6.57 \pm 1.22 \mathrm{a}$ & $5.95 \pm 1.03 \mathrm{ab}$ & $4.18 \pm 1.89 a b c$ & $6.11 \pm 1.30 \mathrm{abc}$ \\
\hline HT: HM 5\% & $6.35 \pm 1.17 \mathrm{a}$ & $6.29 \pm 1.28 \mathrm{ab}$ & $5.84 \pm 1.17 \mathrm{ab}$ & $6.33 \pm 1.32 \mathrm{a}$ & $5.77 \pm 1.41 \mathrm{ab}$ & $4.95 \pm 1.73 a b$ & $6.01 \pm 1.29 \mathrm{abc}$ \\
\hline HHT: HM 7.5\% & $6.40 \pm 1.29 a$ & $5.82 \pm 1.50 \mathrm{ab}$ & $5.60 \pm 1.43 \mathrm{abc}$ & $6.38 \pm 1.15 a$ & $5.76 \pm 1.31 \mathrm{ab}$ & $4.79 \pm 2.09 a b c$ & $5.75 \pm 1.18 \mathrm{abc}$ \\
\hline IHT: HM 10\% & $6.47 \pm 1.21 \mathrm{a}$ & $6.31 \pm 1.26 \mathrm{a}$ & $5.81 \pm 1.67 \mathrm{ab}$ & $6.53 \pm 1.20 \mathrm{a}$ & $5.76 \pm 1.19 a b$ & $5.21 \pm 1.75 a$ & $5.81 \pm 1.27 \mathrm{abc}$ \\
\hline HHT: HS $2.5 \%$ & $6.10 \pm 1.51 \mathrm{ab}$ & $5.48 \pm 1.88 \mathrm{ab}$ & $5.96 \pm 1.12 \mathrm{ab}$ & $6.51 \pm 1.09 a$ & $6.15 \pm 1.52 a$ & $4.64 \pm 2.04 a b c$ & $6.29 \pm 1.27 a$ \\
\hline$H T: H S 5 \%$ & $5.28 \pm 1.52 b c$ & $5.61 \pm 1.50 \mathrm{ab}$ & $5.18 \pm 1.50 \mathrm{bcd}$ & $5.79 \pm 1.34 a b$ & $5.19 \pm 1.50 \mathrm{bc}$ & $4.88 \pm 2.06 a b c$ & $5.03 \pm 1.56 \mathrm{~cd}$ \\
\hline , HT:HS $7.5 \%$ & $5.02 \pm 1.42 c$ & $5.85 \pm 1.43 \mathrm{ab}$ & $4.78 \pm 1.04 \mathrm{~cd}$ & $4.90 \pm 1.51 b c$ & $5.26 \pm 1.75 a b c$ & $3.76 \pm 1.92 \mathrm{c}$ & $4.88 \pm 1.41 d$ \\
\hline IHT: HS $10 \%$ & $4.41 \pm 1.97 c$ & $5.45 \pm 1.64 \mathrm{ab}$ & $4.68 \pm 1.36 \mathrm{~d}$ & $4.65 \pm 1.82 c$ & $4.76 \pm 1.31 c$ & $4.31 \pm 1.90 a b c$ & $4.82 \pm 1.32 \mathrm{~d}$ \\
\hline
\end{tabular}

Nota: $\mathrm{HT}=$ Harina trigo; $\mathrm{HA}=$ Harina avena; $\mathrm{HM}=$ Harina maiz; $\mathrm{HS}=$ Harina sorgo. El \% observado en la tabla indica el grado de sustitución de HT. Medias con letra diferente dentro de la columna expresan diferencias significativas $(p<0.05)$.

Elaboración propia.

al trabajar en la sustitución de harina de trigo por subproductos alimenticios, reportaron resultados de apelmazamiento final en harina de trigo (3413 cP) mayores al obtenido en nuestro estudio.

Otros estudios realizados por Zaidul, Yamauchi, Kim, Hashimoto y Noda (2007), observaron también incrementos significativos en la viscosidad final cuando aumentaron el nivel de suplencia de harina de trigo con almidón en porcentajes de 10 a $50 \%$. Conclusiones atribuidas a fuertes interacciones entre almidón y proteína y al incremento en enlaces disulfuro que pueden ser responsables de altos valores de viscosidad final son a las que llegaron Tadeu Paraginski et al. (2014) para este parámetro de viscosidad. Las propiedades reológicas de las masas son críticas en la manufactura de alimentos, como se lee en Barak, Mugdil, y Khatkar (2013). La masa exhibe un comportamiento viscoelástico, combinando las propiedades de un fluido viscoso y un sólido elástico. La combinación de harina, agua y levadura juega un papel muy importante que define la estructura y propiedades reológicas de la masa, como lo explican Upadhyay, Ghosal, y Mehra (2012).

En cuanto al análisis de TPA, estudios realizados por López y Goldner (2015) sobre la influencia del almacenamiento en pan elaborado con HT y aislado de proteína de altramuz, utilizaron como control el pan elaborado con HT y observaron resultados de cohesividad de 0.80, muy semejante al encontrado en el presente trabajo, además de elasticidad de 0.91, gomosidad de 6.2 y masticabilidad de 5.64. Estudios realizados por Verdú et al. (2015) también utilizando como control HT para elaborar pan, obtuvieron valores muy cercanos a los obtenidos para el estudio en cohesividad 0.9 y elasticidad 1.0; sin embargo, no para dureza, 2.92, gomosidad, 2.6 y masticabilidad, 2.6, que fueron valores algo alejados a los obtenidos en nuestro estudio.

Enlo querespecta a los panes (figura3) sometidos al análisis sensorial, si bien para el parámetro aroma y textura en boca no existieron diferencias cuando se

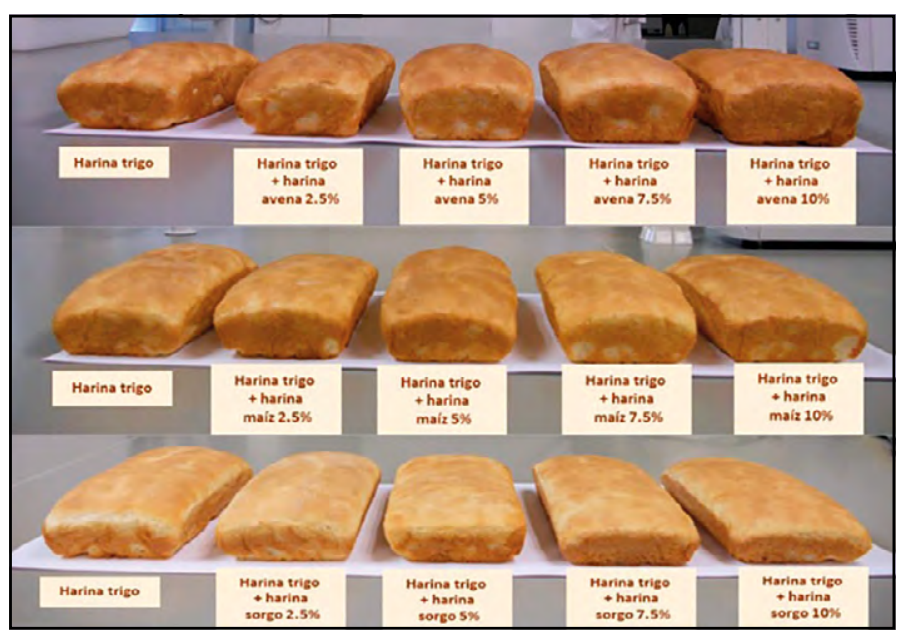

Figura 3. Panes elaborados a partir de harina de trigo sustituida con harina de avena, maíz y sorgo en porcentajes de 2.5, 5, 7.5 y $10 \%$. Fotografía proporcionada por Francisco Vázquez Lara. 
IIVESTIGACIÓn Y CIERCIA DE LA UNIVERSIDAD AUTÓNOMA

utilizó HS en el reemplazo de HT, sí las hubo para el resto de los parámetros, en donde las diferencias más evidentes se observaron cuanto mayor fue el grado de sustitución. Una importante semejanza estadística fue observada en los panes elaborados sólo de HT y en los obtenidos cuando la HT fue sustituida por HA y HM; altos niveles de preferencia en la mayoría de los parámetros analizados por los catadores fueron observados cuando HT fue sustituida por HA, esto mismo sucedió para la sustitución con HM.
CONCLUSIONES

La suplencia de $10 \%$ de harina de trigo con la de avena favoreció el volumen de la masa durante la fermentación. La utilización de harina de maíz o avena a $10 \%$ generó pan más blando con respecto al obtenido sólo de la de trigo, este último muy semejante al elaborado con la de sorgo a $10 \%$. Los panelistas no observaron diferencias entre el pan elaborado sólo de harina de trigo y los obtenidos con la sustitución de $10 \%$ con las de avena o maíz. Los panes con menor calificación fueron los elaborados con harina de sorgo a 10\%; sin embargo, dentro de los parámetros analizados, aroma y textura en boca no mostraron diferencias con respecto al resto de los panes.

REFERENCIAS

- American Association of Cereal Chemists. (2000). Methods 56-11, 76-21. Approved Methods of American Association of Cereal Chemists (10th ed.). St. Paul, MN, US: American Association of Cereal Chemists.

- Barak, S., Mugdil, D., \& Khatkar, B. S. (2013). Relationship of gliadin and glutenin proteins with dough rheology, flour pasting and bread making performance of wheat varieties. LWT-Food Science and Technology, 51 (1), 211-217.

- Batey, I. L. (2007). Interpretation of RVA Curves. In G. B. Crosbie, \& A. S. Ross (Eds.), The RVA Handbook (pp. 19-29). St. Paul, MN: AACC International.

- Berton, B., Scher, J., Villieras, F., \& Hardy, J. (2002). Measurement of hydration capacity of wheat flour: Influence of composition and physical characteristics. Powder Technology, 128(2-3), 326-331.

- Dhingra, S., \& Jood, S. (2002). Organoleptic and nutritional evaluation of wheat breads supplemented with soybean and barley flour. Food Chemistry, 77(4), 479-488.

- Haynes, L. C., Bettge, A. D., \& Slade, L. (2009). Soft wheat and flour products methods review: Solvent retention capacity equation correction. Cereal Foods World, 54, 174-175.

- Kieffer, R., Wieser, H., Henderson, M. H., \& Graveland, A. (1998). Correlations of the breadmaking performance of wheat flour with rheological measurements on a micro-scale. Journal of Cereal Science, 27(1), 53-60.

- Ktenioudaki, A., O’Shea, N., \& Gallagher, E. (2013). Rheological properties of wheat dough supplemented with functional byproducts of food processing: Brewer's spent grain and apple pomace. Journal of Food Engineering, 116(2), 362-368.

- López, E. P., \& Goldner, M. C. (2015). Influence of storage time for the acceptability of bread formulated with lupine protein isolate and added brea gum. LWT-Food Science and Technology, 64(2), 1171-1178.

- Milligan, E. D., Amlie, J. H., Reyes, J., Garcia, A., \& Meyer, B. (1981). Processing for production of edible soy flour. Journal American Oil Chemists' Society, 58(3), 331-333.

- Ram, S., Dawar, V., Singh, R. P., \& Shoran, J. (2005). Application of solvent retention capacity tests for the prediction of mixing properties of wheat flour. Journal of Cereal Science, 42(2), 261-266.

- Shittu, T. A., Raji, A. O., \& Sanni, L. O. (2007). Bread from composite cassava-wheat flour: I. Effect of baking time and temperature on some physical properties of bread loaf. Food Research International, 40(2), 280-290.

- Soleimani Pour Damanab, A. R., Jafary, A., \& Rafiee, Sh. (2011). Monitoring the dynamic density of dough during fermentation using digital imaging method. Journal of Food Engineering, $107(2), 8-13$.

- Tadeu Paraginski, R., Levien Vanier, N., Berrios, J. J., Oliveira, M., \& Cardoso Elias, M. (2014). Physicochemical and pasting properties of maize as affected by storage temperature. Journal of Stored Products Research, 59, 209-214.

- Upadhyay, R., Ghosal, D., \& Mehra, A. (2012). Characterization of bread dough: Rheological properties and microstructure. Journal of Food Engineering, 109(1), 104-113.

- Verdú, S., Vásquez, F., Ivorra, E., Sánchez, A. J., Barat, J. M., \& Grau, R. (2015). Physicochemical effects of chia (Salvia hispanica) seed flour on each wheat bread-making process phase and product storage. Journal of Cereal Science, 65, 67-73.

- Zaidul, I. S. M., Yamauchi, H., Kim, S. J., Hashimoto, N., \& Noda, T. (2007). RVA study of mixtures of wheat flour and potato starches with different phosphorus contents. Food Chemistry, 102(4), 1105-1111. 